J. Clin. Chem. Clin. Biochem.

Vol. 25, 1987, pp. 267-280

(C) 1987 Walter de Gruyter \& Co. Berlin - New York

\title{
Bestimmung der Thromboplastinzeit mit einem neuen standardisierten Thromboplastin aus menschlicher Plazenta: Ergebnisse einer kooperativen Studie
}

\author{
Von M. Barthels \\ Abt. Hämatologie und Onkologie der Med. Hochschule, Hannover
}

H. D. Bruhn

I. Med. Universitätsklinik, Kiel

F. Duckert

Gerinnungslabor des Kantonsspitals, Basel/Schweiz

J. G. Meyer

Zentrallabor des Stadtkrankenhauses Hanau

F. Dati und P. Fuhge

Forschungslaboratorien der Behringwerke AG, Marburg

(Eingegangen am 10. Juli 1986/21. Februar 1987)

Zusammenfassung: An fünf Zentren wurde in Plasmen von gesunden Probanden, von Patienten unter oraler Antikoagulantien-Therapie und von Patienten mit verschiedenen Erkrankungen insbesondere der Leber ein neues empfindliches standardisiertes Thromboplastin aus humaner Plazenta (Thromborel ${ }^{\circledR}$ ) zur Bestimmung der Thromboplastinzeit erprobt. Die Standardisierung dieses Human-Plazenta-Thromboplastins erfolgte durch Vergleichsuntersuchungen mit einer Charge der Referenzpräparation British Comparative Thromboplastin. Der ermittelte internationale Empfindlichkeitsfaktor (ISI) für 14 Chargen des neuen Thromboplastins variiert zwischen 1,04 und 1,29 (Mittelwert: 1,16). Das Reagenz besitzt eine hohe Empfindlichkeit gegenüber allen Faktoren des exogenen Gerinnungssystems und wird von Heparin bis mindestens 0,6 IU/ml nicht beeinflußt. Aus dem Vergleich mit dem British Comparative Thromboplastin Charge Nr. 235 wurde ein therapeutischer Bereich für die stabile Phase der oralen Antikoagulantien-Therapie von 2,4-4,0 ProthrombinRatio bzw. von $15-27 \%$ der Norm èmittelt. Vergleichsuntersuchungen zwischen dem Human-PlazentaThromboplastin und dem British Comparative Thromboplastin an 330 Patienten unter oraler Antikoagulation ergaben eine gute Korrelation sowohl in "Prozent der Norm" als auch in Prothrombin-Ratio.

Determination of prothrombin time with a new standardized thromboplastin from human placenta: Results of a cooperative study

Summary: In five centres a new sensitive standardized thromboplastin from human placenta (Thromborel ${ }^{\circledR S}$ ) for determination of prothrombin time (PT) was evaluated on plasmas from healthy subjects, from patients on oral anticoagulant therapy and from patients with different diseases, especially of the liver. The standardiz-

J. Clin. Chem. Clin. Biochem. / Vol. 25, 1987 / No. 4 
ation of the human placenta thromboplastin (HPT) for prothrombin time determination was performed by comparison with a lot of the Reference Preparation British Comparative Thromboplastin (BCT). The obtained International Sensitivity Index (ISI) for 14 different lots of the new thromboplastin varied between 1.04 and 1.29 (mean value: 1.16 ). The reagent is highly sensitive to the factors of the extrinsic coagulation pathway and is not affected by heparin at least up to $0.6 \mathrm{IU} / \mathrm{ml}$. From the comparison with the British Comparative Thromboplastin lot No. 235, a therapeutical range for the stable phase of the oral anticoagulation of $2.4-4.0$ prothrombin ratio or $15-27 \%$ of normal, respectively, was obtained. Comparison of prothrombin time determination using the Human Placental Thromboplastin and the British Comparative Thromboplastin lot No. 235 in 330 patients on oral anticoagulation showed good correlations either in "percent normal" or in prothrombin ratio.

\section{Einführung}

Thromboplastine sind Lipoproteine, die ubiquitär in Säugetiergeweben vorkommen (1). Im Jahre 1934 führte Quick die Thromboplastine zur Bestimmung der Ein-Phase-Thromboplastinzeit ein (2). Er präparierte einen Acetonextract von Kaninchenhirn als stabile Quelle für Thromboplastin und erstellte mittels einer Verdünnungsreihe von gepooltem Normalplasma eine Referenzkurve (3).

Da der Test einfach war, setzte er sich in zahlreichen Varianten durch, sowohl für die Diagnostik von Gerinnungsstörungen und zur Beurteilung der Leberfunktion als auch für die Überwachung der oralen Antikoagulation mit Vitamin-K-Antagonisten (3).

Zur Bestimmung der Thromboplastinzeit (TPZ, Quicktest, auch Prothrombinzeit, PT) werden Thromboplastine aus verschiedenen Organen (Hirn, Lunge, Plazenta) bzw. Spezies (Kaninchen, Affe, Rind, Mensch) eingesetzt (1). Allerdings sind diese Thromboplastine in ihren spezifischen Eigenschaften nicht vergleichbar und offensichtlich gegen den Mangel verschiedener exogener Gerinnungsfaktoren unterschiedlich empfindlich (4). Außerdem enthalten verschiedene Thromboplastinpräparationen nachträglich zugesetzte Gerinnungsfaktoren wie Fibrinogen und/oder Faktor V oder Heparininhibitoren (4).

Aufgrund dieser Unterschiede lieferte die Bestimmung der Thromboplastinzeit mit verschiedenen Reagenzien (die sogenannten Quicktests) schwer vergleichbare Ergebnisse $(5,6)$. Hinzu kam die Problematik der unterschiedlichen Angaben der im Test erzielten Ergebnisse entweder in "Prozent der Norm" oder als Verlängerung der Gerinnungszeit des Patientenplasmas im Vergleich zu einem Normalplasma (7, 8). Dies alles wirkt sich negativ auf eine Optimierung der Überwachung der oralen Antikoagulantientherapie aus $(7,9)$.

Einen Ausweg aus dieser Situation stellten die Bemühungen verschiedener Gremien zur Standardisierung, der Thromboplastinzeit mit der Bereitstellung von
Referenzthromboplastinen menschlichen Ursprungs $(10,11,12)$ dar. Die erste Referenzpräparation, das Manchester Comparative Reagent (MCR), wurde 1967 aus Menschenhirn isoliert (13). Diese Präparation wurde später in British Comparative Thromboplastin (BCT) umbenannt (14).

Nachdem 1977 die WHO ein Menschenhirn-Thromboplastin (International Reference Preparation of Thromboplastin - IRP 67/40) (15) und 1979 ein Rinderthromboplastin (Ox Brain Thromboplastin OBT 68/434) und ein Kaninchenthromboplastin (Rabbit Brain Thromboplastin RBT 70/178) als Primärstandards anerkannte, wurde 1983 vom WHO Expert Committee on Biological Standardisation ein Modell zur Kalibrierung von Thromboplastinen und somit zur Standardisierung der Thromboplastinzeit unter Verwendung der Referenzpräparationen verabschiedet (16).

In diesem statistischen Modell ist jedes Thromboplastin durch die Steigung seiner Kalibrierungsgeraden, bezeichnet als ISI (= International Sensitivity Index oder Internationaler Empfindlichkeitsfaktor), definiert $(11,17)$. Dieses Modell diente als Grundlage für die Kalibrierung und Standardisierung eines neuen Thromboplastins, das mit einem neuen Herstellungsverfahren aus menschlicher Plazenta gewonnen wird $(18,19)$.

In einer Studie in fünf Zentren wurde die Erprobung des neuen Human-Plazenta-Thromboplastins für die Thromboplastinzeit-Bestimmung an verschiedenen Patientenkollektiven, insbesondere Patienten unter oraler Antikoagulation vorgenommen und gleichzeitig seine Standardisierung und Vergleichbarkeit mit internationalen Referenzpräparationen an einer Charge des British Comparative Thromboplastins geprüft.

\section{Material und Methoden}

Thromboplastine

- Human-Plazenta-Thromboplastin (HPT I): Thromborel ${ }^{\circledR S}$ Behringwerke AG, Marburg :i 


\section{Referenzthromboplastine}

- British Comparative Thromboplastin "BCT" - Herkunft: Menschenhirn - (U.K. Reference Lab. for Anticoagulant Reagents and Control, Manchester, U.K.) Charge Nr. 099 (ISI: 1,048) und Charge Nr. 235 (ISI: 1,039)

\section{Weitere Vergleichsthromboplastine}

- Human-Plazenta-Thromboplastin II (geringer Faktor VIIempfindlich): Calcium-Thromboplastin (Behringwerke AG, Marburg)

- Human-Plazenta-Thromboplastin III (nicht standardisiert): Thromborel® (Behringwerke AG, Marburg)

- Menschenhirnthromboplastin (MHT) (Basel)

- Kaninchen-Hirn-Thromboplastin: Thromboplastin FS (Merz + Dade, München)

- Kaninchen-Lunge/-Hirn-Thromboplastin: Simplastin ${ }^{\circledR}$ (Goedecke, Freiburg)

- Kaninchen-/Affenhirn-Thromboplastin: Thromboplastin "a" (Boehringer Mannheim GmbH, Mannheim).

\section{Andere Reagenzien}

\section{Frischpoolplasma}

Gepooltes Plasma, gewonnen aus frischen Plasmaproben von 150 männlichen Spendern (Alter 18-40 Jahre). In den einzelnen Plasmaproben wurden die Thromboplastinzeit und die partielle Thromboplastinzeit bestimmt. Die Plasmaproben wurden für den Pool verwendet, nur wenn die Thromboplastinzeit einen Wert von $>70 \%$ der Norm und die partielle Thromboplastinzeit einen Wert < $42 \mathrm{~s}$ aufwiesen. Das Poolplasma wurde portioniert und die Aliquots wurden bis zur Verwendung bei $-70^{\circ} \mathrm{C}$ gelagert.

\section{Normalplasma}

Standard-Human-Plasma (Behringwerke AG, Marburg)

\section{Heparin}

Liquemin ${ }^{\circledR} 5000 \mathrm{IU} / \mathrm{ml}$ (Hoffman-La Roche, Grenzach)

Calciumchlorid-Lösung $0,025 \mathrm{~mol} / \mathrm{l}$.

Natriumcitrat-Lösung $0,11 \mathrm{mmol} / 1$.

\section{Geräte}

Die Bestimmung der Thromboplastinzeit wurde entweder manuell (Häkchen-Methode) oder mechanisch mit folgenden $\mathrm{Ge}-$ räten durchgeführt: Kugel-Coagulometer KC 10 und Coagulometer nach Schnitger \& Gross (beide Fa. Amelung, Lemgo).

\section{Plasmagewinnung}

Die Blutentnahme erfolgte in Spritzen, die in der Vorlage ungepufferte Natriumcitrat-Lösung $0,11 \mathrm{~mol} / 1$ enthielten. Das Verhältnis Antikoagulanz/Blut betrug 1 Teil zu 9 Teilen. Das Blut wurde nach Entnahme $10 \mathrm{~min}$ bei $1500 \mathrm{~g}$ zentrifugiert, das überstehende Plasma entnommen und entweder sofort verarbeitet oder nạch Schockgefrieren bei $-70^{\circ} \mathrm{C}$ aufbewahrt. Die eingefrorenen Plasmen wurden bei $+37^{\circ} \mathrm{C}$ aufgetaut. Lyophilisierte Plasmẹn wurden nach Angabe des Herstellers aufgelöst.

Durchführung der Thromboplastinzeit-Bestimmung Human-Plazenta-Thromboplastin I (HPT I)

Das Thromboplastin-Reagenz wurde mit dest. Wasser gelöst und vor Gebrauch mindestens $15 \mathrm{~min}$ bei $+37^{\circ} \mathrm{C}$ inkubiert.
Testansatz: In auf $+37^{\circ} \mathrm{C}$ vorgewärmte Glas- bzw. Kunststoffröhrchen wurden $0,1 \mathrm{ml}$ Plasma pipettiert, $1 \mathrm{~min}$ bei $+37^{\circ} \mathrm{C}$ inkubiert und dann $0,2 \mathrm{ml}$ Reagenz (auf $+37^{\circ} \mathrm{C}$ temperiert) zugegeben.

Mit der Zugabe des Reagenzes wurde die Meßstelle am Coagulometer bzw. die Stoppuhr gestartet und die Gerinnungszeit bestimmt.

\section{British Comparative Thromboplastin (BCT)}

Die Abfullung des British Comparative Thromboplastin Charge Nr. 235 wurde in $1 \mathrm{ml}$ Rekonstitutionslösung gelöst und $5 \mathrm{~min}$ bei Raumtemperatur stehen gelassen (20).

Testansatz: In auf $+37^{\circ} \mathrm{C}$ vorgewärmte Glas- bzw. Kunststoffröhrchen wurden $0,1 \mathrm{ml}$ Reagenz und $0,1 \mathrm{ml}$ Plasma pipettiert und $1 \mathrm{~min}$ bei $+37^{\circ} \mathrm{C}$ inkubiert, danach $0,1 \mathrm{ml}$ Calciumchlorid-Lösung $0,025 \mathrm{~mol} / 1$ (auf $+37^{\circ} \mathrm{C}$ temperiert) zugegeben. Mit Zugabe der Calciumchlorid-Lösung wurde die Gerinnungszeit bestimmt.

\section{Andere Thromboplastine}

Die Durchführung der Thromboplastinzeit-Bestimmung erfolgte wie bei dem Human-Plazenta-Thromboplastin I. Sämtliche Bestimmungen der Thromboplastinzeit wurden immer als Doppelbestimmungen durchgeführt. Es wurde immer der Mittelwert der Doppelbestimmung für die weitere Berechnung verwendet. Im Falle von größeren Abweichungen bei der Doppelbestimmung wurde die Bestimmung wiederholt.

\section{Darstellung der Ergebnisse}

Die Darstellung der Ergebnisse erfolgt in Sekunden (s), in "Prozent der Norm" (\% der Norm) oder als ProthrombinRatio.

\section{Berechnung der Prozent-Werte anhand einer Bezugskurve}

Die gemessenen Gerinnungszeiten wurden anhand einer Bezugskurve in Prozent-Werte (prozentuale Verdünnung eines "normalen" Plasmas: "Prozent der Norm") umgerechnet. Die Erstellung der Bezugskurven für die Thromboplastinzeit-Bestimmung mit den verschiedenen Thromboplastinen erfolgte mit einem Frischpoolplasma bzw. mit einem Standard-HumanPlasma entweder unverdünnt oder durch Verdünnen mit isotoner Natriumchloridlösung im Verhältnis $1: 2,1: 4$ und $1: 8$. Die Gerinnungszeiten des Normalplasmas bzw. dessen Verdünnuingen werden den Reziprokwerten der jeweiligen prozentualen Verdünnung zugeordnet. In Abbildung 1 sind die Thromboplastinzeit-Bezugskurven für das Human-Plazenta-Thromboplastin I, für das British Comparative Thromboplastin und für ein herkömmliches Thromboplastin, das eine geringere Faktor-VIIEmpfindlichkeit besitzt, dargestellt.

\section{Prothrombin-Ratio (PR)}

Die Prothrombin-Ratios (oder Thromboplastinzeit-Quotienten) werden aus den Quotienten der Gerinnungszeit der untersuchten Plasmen und der Gerinnungszeit eines in der Versuchsreihe eingesetzten Normal- bzw. Referenzplasmas errechnet. Dieser Quotient ist im Falle, daß das Patientenplasma die gleiche Gerinnungszeit wie das Normalplasma hat, 1,0 und im Falle einer auf das Doppelte verlängerten Gerinnungszeit 2,0. Die Werte in Prothrombin-Ratio erlauben unter Berücksichtigung des für die betreffende Thromboplastincharge spezifischen "Empfindlichkeitsfaktors" aus der Gleichung INR = PR ${ }^{\text {ISI }}$ die Thromboplastinzeit eines oral antikoagulierten Patienten in international gültiger "normalisierter" Prothrombin-Ratio („International Normalized Ratio" = "INR") umzurechnen $(11,21)$ (Tab. 1). 


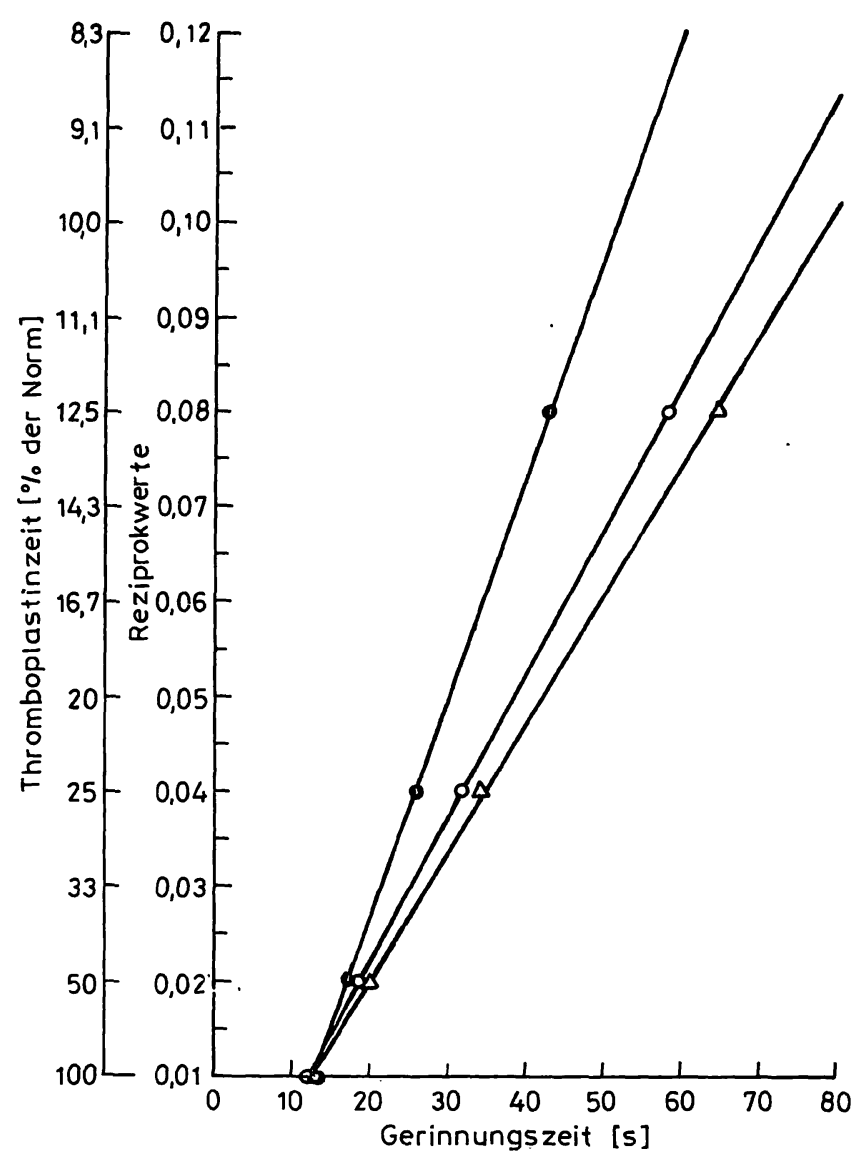

Abb. 1. Bezugskurven für die Thromboplastinzeit in \% der Norm für ein Normalplasma unter Verwendung verschiedener Thromboplastine.

o-o Human-Plazenta-Thromboplastin

- - Human-Plazenta-Thromboplastin, geringer

Faktor VII-empfindlich

$\Delta-\Delta$ British Comparative Thromboplastin

Die Gerinnungszeiten des Normalplasmas (Abszisse) werden den Reziprokwerten der Verdünnungen (Ordinate) zugeordnet.

Bestimmung der Faktorenempfindlichkeit (Faktor II, V, VII, X)

Die Einstellung unterschiedlicher Faktorenkonzentrationen (100, 50, 20, 5, $1 \%$ der Norm) erfolgte durch Mischmengen von tiefgefrorenem normalen Plasmapool mit dem jeweiligen Faktoren-Mangelplasma in folgenden Verhältnissen: $1 \mathrm{ml} / 0 ; 0,5$ $\mathrm{ml} / 0,5 \mathrm{ml} ; 0,2 \mathrm{ml} / 0,8 \mathrm{ml} ; 0,05 \mathrm{ml} / 0,95 \mathrm{ml} ; 0,01 \mathrm{ml} / 0,99 \mathrm{ml}$.

Ạn diesen Plasmen wurde die Thromboplastinzeit unter Verwendung verschiedener Thromboplastin-Reagenzien bestimmt und die Werte in Prothrombin-Ratio ausgedruckt.

\section{Bestimmung der Heparinempfindlichkeit}

Die Testung der Heparin-Empfindlichkeit erfolgte durch Bestimmung der Thromboplastinzeit in verschiedenen Abfüllungen eines Standard-Human-Plasmas, das mit jeweils $1 \mathrm{ml}$ von verschiedenen Heparinlösungen aufgelöst wurde. Diese Lösungen wurden durch Verdünnung einer Heparinlösung (Heparin $5000 \mathrm{IU} / \mathrm{ml}$ ) mit dest. Wasser erhalten. Sie enthielten Heparinkonzentrationen von 0,1 bis $1,0 \mathrm{IU} / \mathrm{ml}$.

\section{Untersuchungsgut}

Im Rahmen der kooperativen Studie wurden Plasmaproben von 139 gesunden Probanden, 161 Patienten mit verschiedenen Erkrankungen, davon 40 Patienten mit Lebererkrankungen und 330 Patienten unter oraler Antikoagulation untersucht.
Tab. 1. Wertetabelle für eine typische Charge des HumanPlazenta-Thromboplastins.

Charge Nr.: 505429

Werte für Schnitger/Gross

\begin{tabular}{|c|c|c|c|}
\hline $\begin{array}{l}\% \\
\text { der } \\
\text { Norm }\end{array}$ & $\begin{array}{l}\text { Thrombo- } \\
\text { plastinzeit } \\
\text { (s) }\end{array}$ & $\begin{array}{l}\text { Prothrom- } \\
\text { bin-Ratio } \\
\text { (PR) }\end{array}$ & $\begin{array}{l}\text { International } \\
\text { Normalized } \\
\text { Ratio (INR) }\end{array}$ \\
\hline 100 & 12,1 & 1,00 & 1,01 \\
\hline 95 & 12,5 & 1,03 & 1,03 \\
\hline 90 & 12,8 & 1,06 & 1,07 \\
\hline 85 & 13,3 & 1,09 & 1,10 \\
\hline 80 & 13,7 & 1,13 & 1,15 \\
\hline 75 & 14,3 & 1,18 & 1,21 \\
\hline 70 & 14,9 & 1,23 & 1,27 \\
\hline 65 & 15,6 & 1,29 & 1,34 \\
\hline 60 & 16,4 & 1,36 & 1,42 \\
\hline 55 & 17,4 & 1,44 & 1,52 \\
\hline 50 & 18,6 & 1,53 & 1,63 \\
\hline 48 & 19,1 & 1,58 & 1,69 \\
\hline 46 & 19,7 & 1,63 & 1,75 \\
\hline 44 & 20,3 & 1,68 & 1,82 \\
\hline 42 & 21,0 & 1,74 & 1,89 \\
\hline 40 & 21,8 & 1,80 & 1,97 \\
\hline 38 & 22,7 & 1,87 & 2,05 \\
\hline 36 & 23,6 & 1,95 & 2,16 \\
\hline 34 & 24,7 & 2,03 & 2,26 \\
\hline 32 & 25,8 & 2,13 & 2,39 \\
\hline 30 & 27,2 & 2,24 & 2,53 \\
\hline 29 & 27,9 & 2,30 & 2,61 \\
\hline 28 & 28,7 & 2,37 & 2,70 \\
\hline 27 & 29,6 & 2,44 & 2,79 \\
\hline 26 & 30,5 & 2,52 & 2,89 \\
\hline 25 & 31,5 & 2,60 & 3,00 \\
\hline 24 & 32,6 & 2,69 & 3,12 \\
\hline 23 & 33,7 & 2,78 & 3,24 \\
\hline 22 & 35,0 & 2,89 & 3,39 \\
\hline 21 & 36,4 & 3,00 & 3,54 \\
\hline 20 & 37,9 & 3,13 & 3,71 \\
\hline 19 & 39,6 & 3,27 & 3,91 \\
\hline 18 & 41,5 & 3,43 & 4,13 \\
\hline 17 & 43,6 & 3,60 & 4,36 \\
\hline 16 & 46,0 & 3,80 & 4,64 \\
\hline 15 & 38,7 & 4,02 & 4,95 \\
\hline 14 & 51,8 & 4,27 & 5,31 \\
\hline 13 & 55,3 & 4,56 & 5,73 \\
\hline 12 & 59,5 & 4,91 & 6,23 \\
\hline 11 & 64,4 & 5,31 & 6,82 \\
\hline 10 & 70,2 & 5,79 & 7,53 \\
\hline 9 & 77,4 & 6,39 & 8,44 \\
\hline 8 & 86,4 & 7,13 & 9,57 \\
\hline 7 & 97,9 & 8,08 & 11,05 \\
\hline 6 & 113,3 & 9,34 & 13,06 \\
\hline 5 & 134,8 & 11,21 & 15,96 \\
\hline
\end{tabular}

International Sensitivity Index (ISI) 1,15

\section{Ergebnisse} Präzision der Thromboplastinzeit-Bestim-
mung

Die Reproduzierbarkeit in der Serie wurde an sechs unterschiedlichen Chargen des neuen Human-Plazenta-Thromboplastins unter Vêrwendung verschiedener Verdünnungen eines Frischplasmapools und verschiedener Kontrollplasmen in normalem und pathologi- 
schem Bereich untersucht. Für die Präzision in der Serie (Intraassay-Varianz) wurden Variationskoeffizienten zwischen 0,4 und 3,0\% für normale Plasmen und zwischen 0,8 und $5,3 \%$ für pathologische Plasmen im Bereich zwischen 25 und $10 \%$ der Norm ermittelt.

Die Präzision von Tag zu Tag (Interassay-Varianz) ergab für die Thromboplastinzeit-Bestimmung (Mittelwert aus sechs Bestimmungen) an vier verschiedenen Tagen Variationskoeffizienten zwischen 1,7 und $2,4 \%$ für normale Plasmen und zwischen 2,2 und $3,7 \%$ für pathologische Plasmen im Bereich zwischen 25 und $10 \%$ der Norm.

\section{Chargenkonstanz}

Die Reproduzierbarkeit von Charge zu Charge (14 verschiedene Chargen) wurde unter Verwendung eines von 150 gesunden Männern gewonnenen und bis zum Zeitpunkt der Untersuchungen bei $-70 /$ $-80^{\circ} \mathrm{C}$ tiefgefrorenen Plasmapools untersucht (Ergebnisse des Zentrums D). Es ergaben sich Variationskoeffizienten zwischen 2,2 und 3,3\% für Thromboplastinzeit-Werte zwischen 100 und 10 "Prozent der Norm" (Tab. 2).
Tab. 2. Chargenkonstanz des Human-Plazenta-Thromboplastins (14 verschiedene Chargen).

\begin{tabular}{llll}
\hline & Mittelwert & Bereich & $\begin{array}{l}\text { Variations- } \\
\text { koeffizient }\end{array}$ \\
Verdünnung & $\begin{array}{l}\text { Thrombo- } \\
\text { plastinzeit } \\
(\mathrm{s})\end{array}$ & $\begin{array}{l}\text { Thromboplastin- } \\
\text { zeit }\end{array}$ & $(\mathrm{s})$ \\
\hline 100 & 11,8 & $11,0-12,3$ & 2,7 \\
50 & 18,0 & $17,2-18,6$ & 2,2 \\
25 & 30,5 & $29,4-31,7$ & 2,6 \\
10 & 68,0 & $65,3-71,5$ & 3,3 \\
\hline ISI & 1,16 & $1,04-1,29$ & 4,9 \\
\hline
\end{tabular}

\section{Faktoren-Empfindlichkeit}

Bei der Bestimmung der Empfindlichkeit von verschiedenen Thromboplastinen bei Mangel der Gerinnungsfaktoren II, V, VII und X (4) zeigte sich, daß das hier beschriebene Human-Plazenta-Thromboplastin eine hohe Empfindlichkeit für alle Faktoren des exogenen Gerinnungssystems einschließlich Faktor VII besitzt (Ergebnisse des Zentrums D) (Abb. 2).
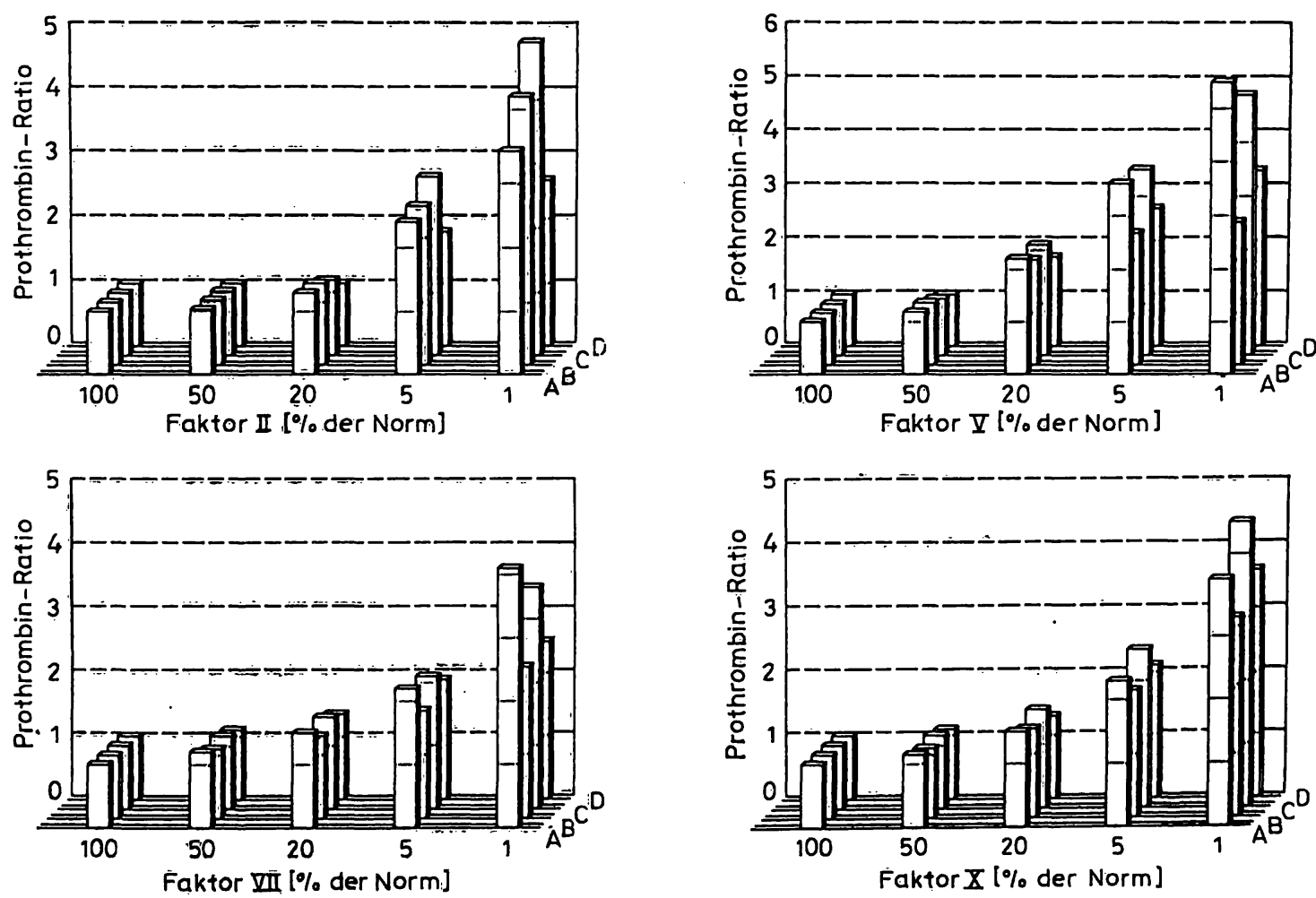

Abb. 2. Faktorenempfindlichkeit für verschiedene Thromboplastine. Aufgetragen ist die Zeitverlängerung (Prothrombin-Ratio) durch die jeweilige Faktorenverminderung $(50,20,5,1 \%$ der Norm) gegenüber Normalplasma (100 \% der Norm).

Thromboplastin aus:

$A=$ Human-Plazenta

$\mathrm{B}=$ Kaninchenlunge/-hirn

$\mathrm{C}=\mathrm{Kaninchenhirn}$

$\mathrm{D}=$ Kaninchen-/Affenhirn

J. Clin. Chem. Clin. Biochem. / Vol. 25, 1987 / No. 4 
Der Vergleich zwischen Thromboplastinen aus Human-Plazenta, aus einer Mischung von Kaninchenlunge und -hirn, aus Kaninchenhirn und aus einer Mischung von Kaninchen- und Affenhirn ergab, daß das neue Human-Plazenta-Thromboplastin empfindlicher für die Faktoren V und VII ist als alle anderen untersuchten Thromboplastine. Bei der Faktor XEmpfindlichkeit zeigte sich nur das Thromboplastin aus Kaninchenhirn empfindlicher als das HumanPlazenta-Thromboplastin. Das Human-PlazentaThromboplastin ist auch dem Referenzthromboplastin British Comparative Thromboplastin aus $\mathrm{Hu}-$ manhirn in der Faktorenempfindlichkeit vergleichbar.

\section{Heparineinflu $B$}

Bei der Thromboplastinzeit-Bestimmung in Kontrollplasmen, die Heparinkonzentrationen zwischen 0,1 und $1,0 \mathrm{IU} / \mathrm{ml}$ enthielten, konnte festgestellt werden, $\mathrm{da} ß$ die gemessenen Thromboplastinzeit-Werte erst bei Heparinkonzentrationen von $>0,6 \mathrm{IU} / \mathrm{ml}$ beeinflußt werden und die Interventionsgrenze von 1,2 Prothrombin-Ratio überschreiten (Ergebnisse des Zentrums D).

\section{Ermittlung der Referenzwerte}

Die Referenzwerte für die Thromboplastinzeit-Bestimmung mittels zweier Chargen des untersuchten Human-Plazenta-Thromboplastins wurden an 132 gesunden männlichen Probanden im Alter zwischen 18 und 35 Jahren ermittelt.

Der Referenzbereich (22) (jeweils 2,5. bis 97,5. Perzentile) betrug 10,9-13,3 s (Medianwert: 12,0 s) (Abb. 3) bzw. 131-85\% der Norm bzw. 0,89-1,08 Prothrombin-Ratio.

Als Referenzwerte können Thromboplastinzeit-Werte $<14$ s bzw. $>80 \%$ der Norm bzw. < 1,15 Prothrombin-Ratio betrachtet werden. Im Hinblick auf die Entscheidung, ob medizinische Konsequenzen folgen müssen, kann die Interventionsgrenze bei einem Thromboplastinzeit-Wert $<75 \%$ der Norm bzw. $>$ 1,2 Prothrombin-Ratio festgelegt werden.

\section{Überwachung der oralen Antikoagulatien- therapie}

Das Human-Plazenta-Thromboplastin wurde zur Bestimmung der Thromboplastinzeit bei 32 Patienten sowohl in der Einstellungsphase als auch in der stabilen Phase der Therapie mit Vitamin K-Antagonisten verwendet, wie von Loeliger et al. (23) empfohlen. In den Plasmen dieser Patienten wurde gleichzeitig die

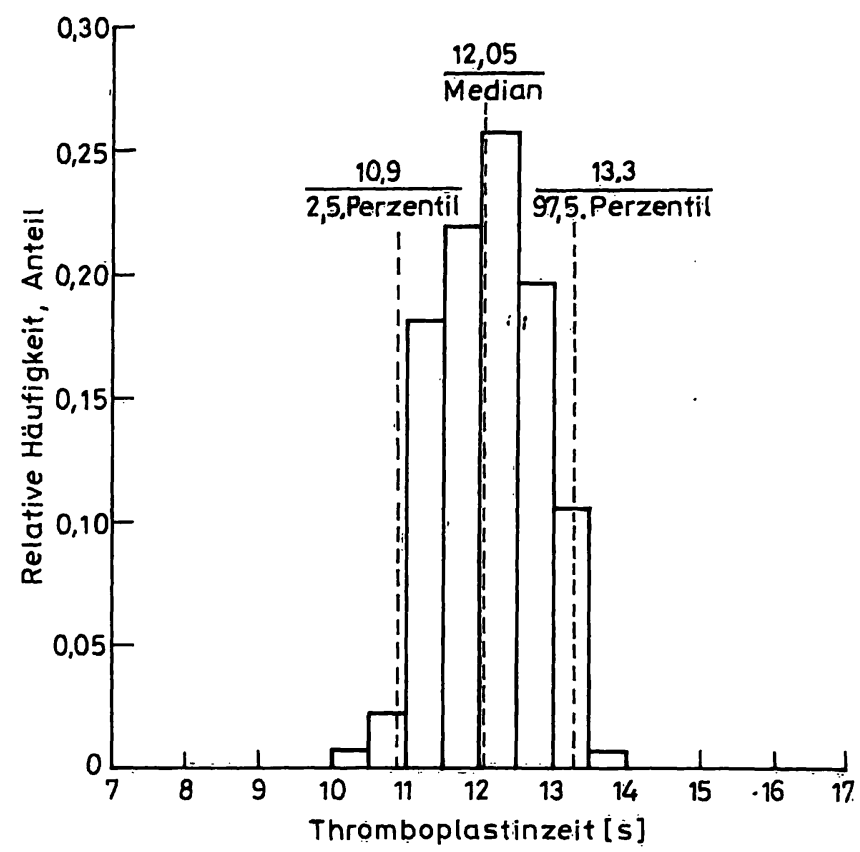

Abb. 3. Häufigkeitsverteilung der Sekundenwerte für die Thromboplastinzeit-Bestimmung mit dem Human-Plazenta-Thromboplastin in einem Kollektiv von 132 gesunden Probanden.

Thromboplastinzeit-Bestimmung mit verschiedenen Thromboplastinen (u.a. mit den Human-PlazentaThromboplastinen I, II bzw. III und mit dem British Comparative Thromboplastin) und die Bestimmung der Faktor II-, Faktor VII- und Faktor X-Aktivitäten vorgenommen. In Abbildung 4 ist die Verlaufskontrolle der Einstellungsphase von einem Patienten bei oraler Antikoagulation wiedergegeben.

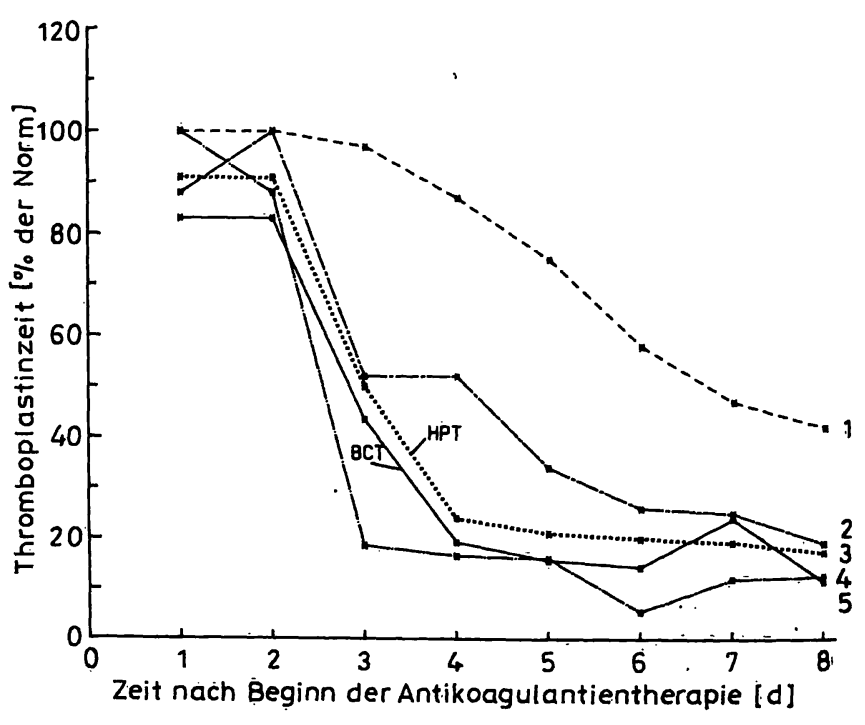

Abb. 4. Thromboplastinzeit-Werte (\% der Norm) für das $\mathrm{Hu}$ man-Plazenta-Thromboplastin und für das British Comparative Thromboplastin und Faktorenaktivitäten während der Einstellung auf orale Antikoagulantien.

1: Faktor II

2: Faktor X

3: Human-Plazenta Thromboplastin I (HPT)

4: Faktor VII

5: British Comparative Thromboplastin (BCT)

J. Clin. Chem. Clin. Biochem. / Vol. 25, 1987 / No. 4 
Der zeitliche Verlauf der Thromboplastinzeit-Werte mit Human-Plazenta-Thromboplastin und mit British Comparative Thromboplastin spiegelt besonders die Aktivitäten der Faktoren VII und X wider.

Die Empfindlichkeit verschiedener Thromboplastine läßt sich bei der Gegenüberstellung der verschiedenen Thromboplastinzeit-Werte im Verlauf der oralen Antikoagulantien-Therapie besonders hervorheben, wenn die Ergebnisse der Thromboplastinzeit-Bestimmung als Prothrombin-Ratio angegeben werden. Als Beispiel ist der Verlauf in Abbildung 5 wiedergegeben.

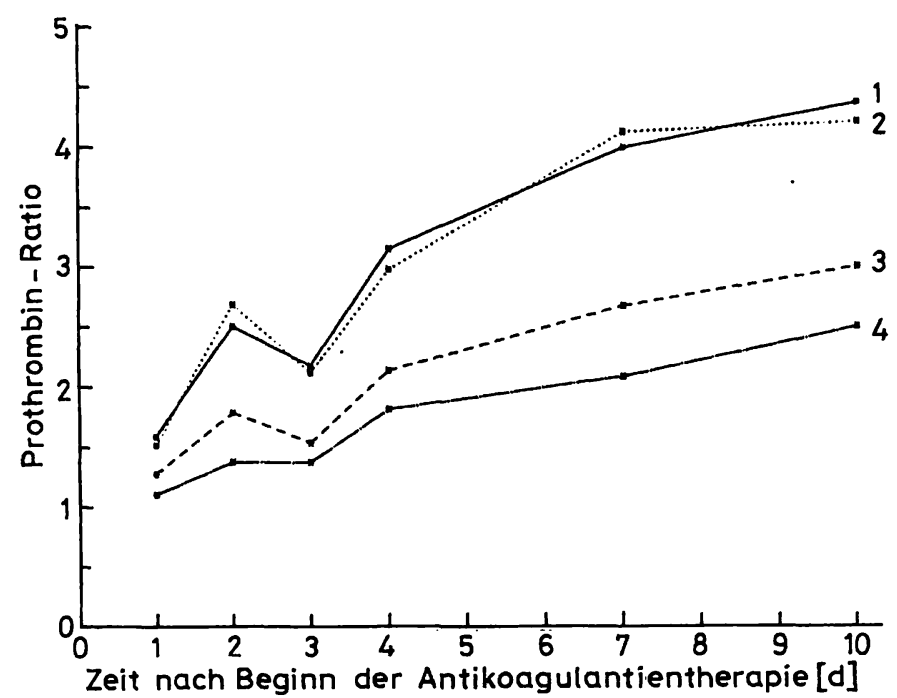

Abb. 5. Verhalten der Thromboplastinzeit-Werte (Prothrombin-Ratio) mittels verschiedener Thromboplastine mit unterschiedlicher Empfindlichkeit während der Einstellphase der oralen Antikoagulation.

1: Human-Plazenta-Thromboplastin I

2: British Comparative Thromboplastin

3: Human-Plazenta-Thromboplastin III (nicht standardisiert)

4: Human-Plazenta-Thromboplastin II (weniger F VIIempfindlich)

Thromboplastinżeit-Bestimmung bei Patienten mit verschiedenen Erkrankungen (u. a. Lebererkrankungen)

Die Thromboplastinzeit wurde auch in Plasmen von 161 Patienten, die nicht unter Antikoagulantien-Therapie standen, insbesondere mit Lebererkrankungen bestimmt. Gleichzeitig wurde in 20 der Patienten die Bestimmung der Faktor II-, Faktor VII- und Faktor $\mathrm{X}$-Aktivität vorgenommen (Abb. 6). Bei der Wiedergabe der Ergebnisse von fünf Patienten mit Lebererkrankungen wurden die mit dem Human-PlazentaThromboplastin erzielten Thromboplastinzeit-Werte und die Einzelfaktorenaktivitäten den Mittelwerten der entsprechenden Aktivitäten gegenübergestellt. Die letzteren zeigten eine gute Korrelation mit den gemessenen Thromboplastinzeit-Werten.

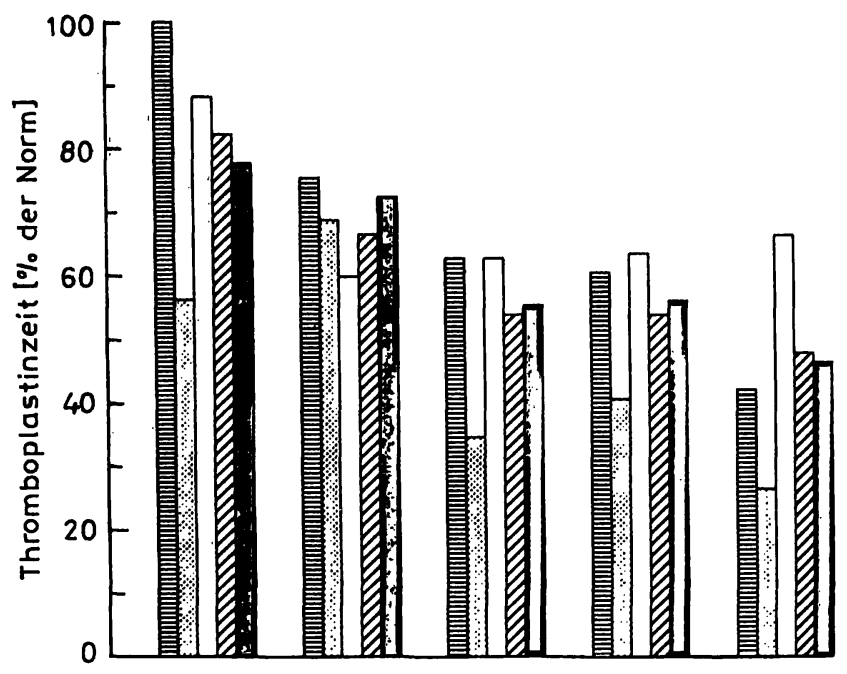

Abb. 6. Thromboplastinzeit-Werte mit dem Human-PlazentaThromboplastin und Faktorenaktivitäten bei Patienten mit Lebererkrankungen. Der Mittelwert aus den Aktivitäten der Gerinnungsfaktoren II, VII und $X$ ist den gemessenen Thromboplastinzeit-Werten gegenübergestellt.

Faktor II

Faktor VII

Faktor X .

Mittel von F II + F VII + F X

Human-Plazenta-Thromboplastin 1

Vergleichsuntersuchungen zwischen verschiedenen Thromboplastinen bei Patienten unter Antikoagulantien-Therapie

Im Rahmen der kooperativen Studie wurden in den fünf Zentren Vergleichsuntersuchungen der Thromboplastinzeitbestimmung mit dem Human-PlazentaThromboplastin und mit dem Referenzthromboplastin British Comparative Thromboplastin Charge Nr. 235 unter Verwendung von 330 Plasmen von oral antikoagulierten Patienten durchgeführt.

Der Vergleich der mit beiden Thromboplastinen erzielten Thromboplastinzeit-Werte unter Hinzuziehen der statistischen Methode der linearen Regression ergab eine gute Korrelation sowohl für die Werte in „\% der Norm“ ( $y=0,97 x-1,6 ; r=0,979)$ (Abb. 7) als auch in Prothrombin-Ratio $(y=1,034 x \pm 0$; $r=0,964)(A b b .8)$.

Auch die Auswertung der Daten mit dem nicht-parametrischen Verfahren nach Passing \& Bablok $(24,25)$ ergab über den gesamten Bereich eine hohe Übereinstimmung der Thromboplastinzeit-Werte beider Methoden (Abb. 9).

Die Ergebnisse der Vergleichsuntersuchungen haben gezeigt, daß zwischen dem Human-Plazenta-Thromboplastin und dem British Comparative Thromboplastin Charge Nr. 235 weiterhin eine gute Korrelation 


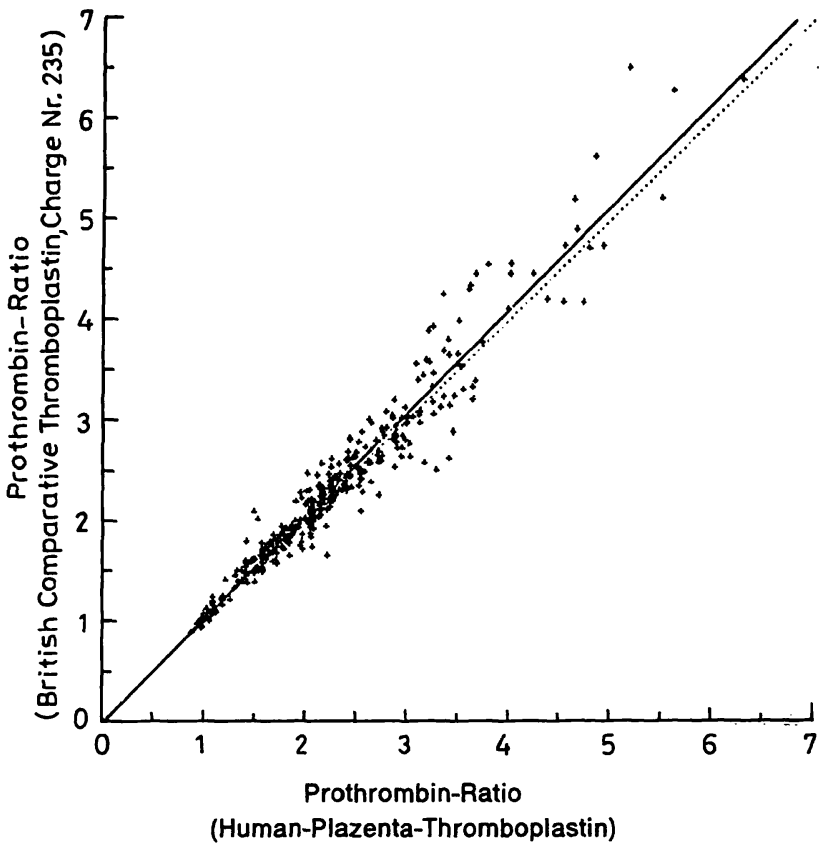

Abb. 7. Vergleich von Thromboplastinzeit-Bestimmungen mit dem Human-Plazenta-Thromboplastin und dem British Comparative Thromboplastin für das Gesamtkollektiv von Patienten unter oraler Antikoagulation $(n=330)$ auf der Basis von Werten in \% der Norm. Auswertung nach der Methode der linearen Regression:

$r=0,964 ; y=1,03 x \pm 0$.

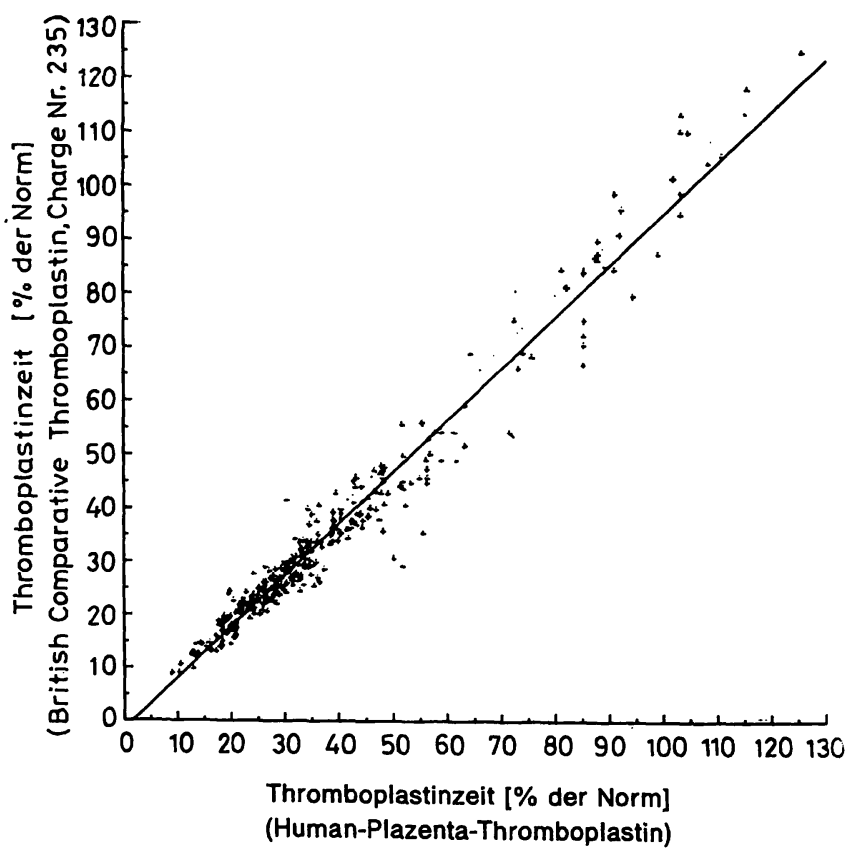

Abb. 8. Vergleich von Thromboplastinzeit-Bestimmungen mit dem Human-Plazenta-Thromboplastin und dem British Comparative Thromboplastin für das Gesamtkollektiv von Patienten unter oraler Antikoagulation $(n=330)$ auf der Basis von Werten in ProthrombinRatio.

Auswertung nach der Methode der linearen Regression:

$r=0,979 ; y=0,97 x-1,6$.

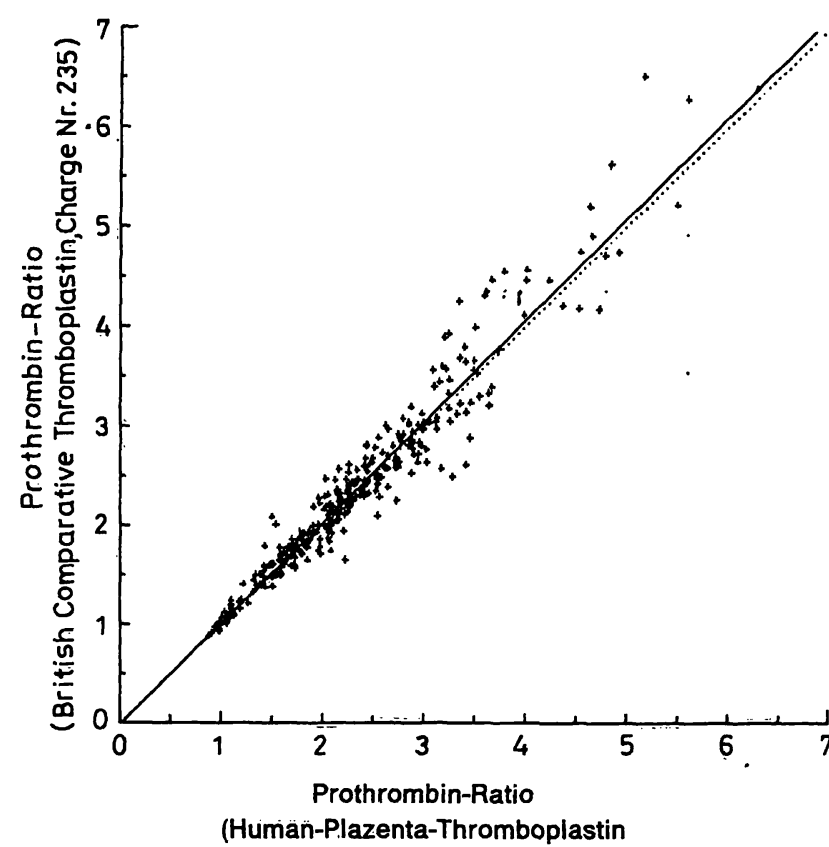

Abb. 9. Vergleich von Thromboplastinżeit-Bestimmungen mit dem Human-Plazenta-Thromboplastin I und dem British Comparative Thromboplastin für das Gesamtkollektiv von Patienten unter oraler Antikoagulation $(n=330)$ auf der Basis von Werten in ProthrombinRatio.

Auswertungsmethode nach Passing \& Bablok (24): $y=1,02 x-0,02$

Linearität: Ja.

Keine signifikante Abweichung der Steigung von 1: 0,99 bis 1,05 .

Keine signifikante Abweichung der Steigung des yAchsenabschnitts von 0 : $-0,07$ bis 0,03 .

besteht, auch wenn die Prothrombin-Ratio-Werte der Patienten getrennt in Einstellungsphase und in stabiler Phase (Prothrombin-Ratio zwischen 1,5 und 5,0) der oralen Antikoagulantien-Therapie für beide Methoden getrennt verglichen werden (Abb. 10).

Die gute Korrelation zwischen dem Human-PlazentaThromboplastin und Menschenhirn-Thrombokinasen, die bei den Vergleichsuntersuchungen mit dem British Comparative Thromboplastin festgestellt wurde, wurde auch bei weiteren an 80 Patienten durchgeführten Untersuchungen zwischen dem $\mathrm{Hu}$ man-Plazenta-Thromboplastin und einer in einem der fünf Zentren verwendeten Menschenhirn-Thrombokinase (MHT) bestätigt (Abb. 11). Als Korrelationskoeffizient wurde ein Wert von 0,989 ermittelt. Die Regressionsgerade wird durch die Gleichung y $(\mathrm{MHT})=0,99(\mathrm{HPT})-0,2$ beschrieben.

\section{Überprüfung der Standardisierung des $\mathrm{Hu}$ -} man-Plazenta-Thromboplastins

Gemäß einer Empfehlung der Weltgesundheitsorganisation (WHO) $(21,26)$ wird jede hergestellte Charge 


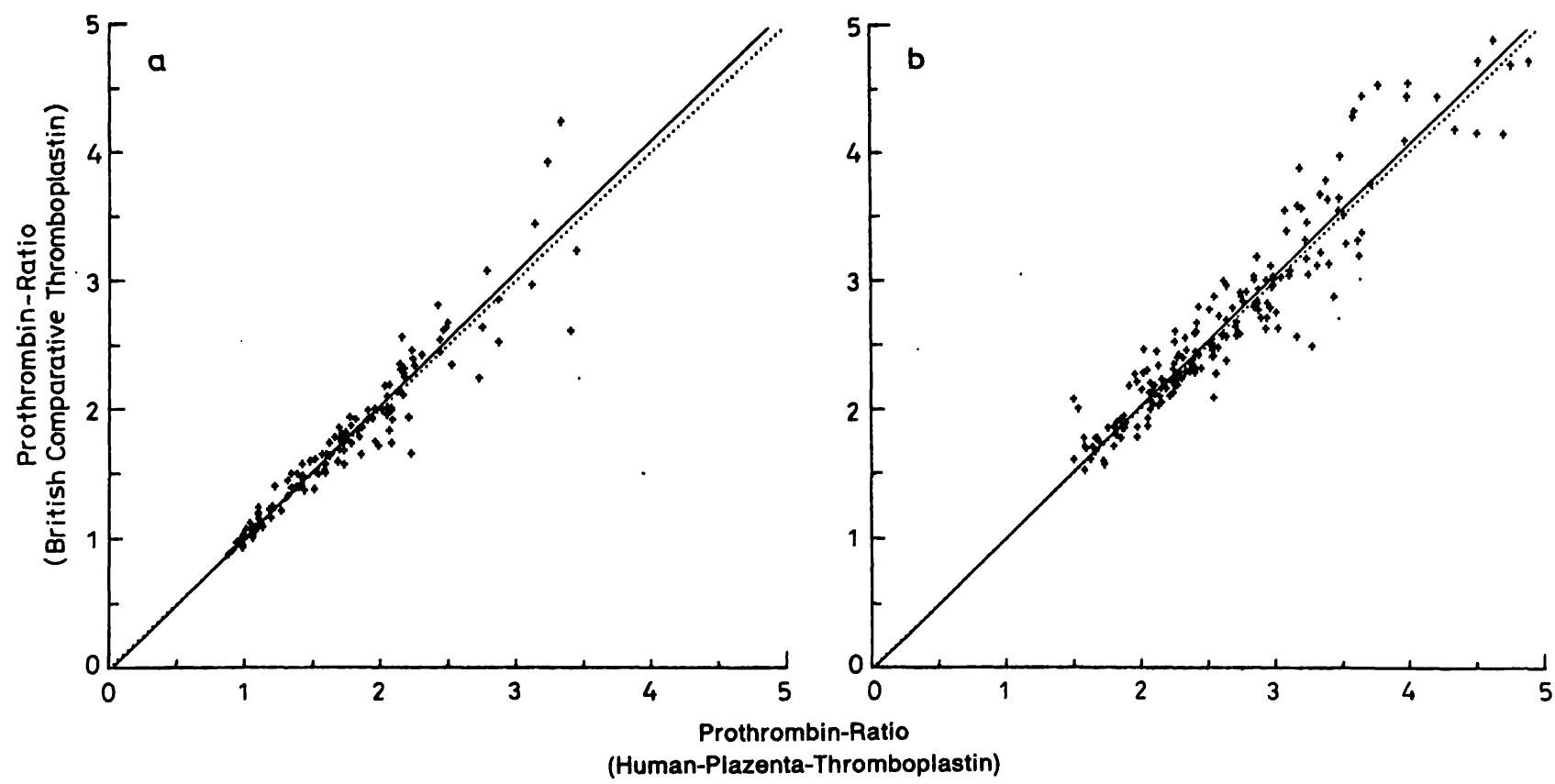

Abb. 10. Thromboplastinzeit-Bestimmung mittels dem Human-Plazenta-Thromboplastin und dem British Comparative Thromboplastin. Korrelation der Prothrombin-Ratio-Werte von Patienten (a) in der Einstellungsphase $(n=132)$ und $(b)$ in der stabilen Phase $(n=184)$ der oralen Antikoagulation. Auswertungsmethode nach Passing \& Bablok (24).

Einstellphase: $\mathrm{y}=1,03 \mathrm{x}-0,03$;

stabile Phase: $y=1,02 x-0,02$;

keine signifikante Abweichung der Steigungen von 1 und der $y$-Achsenabschnitte von 0.

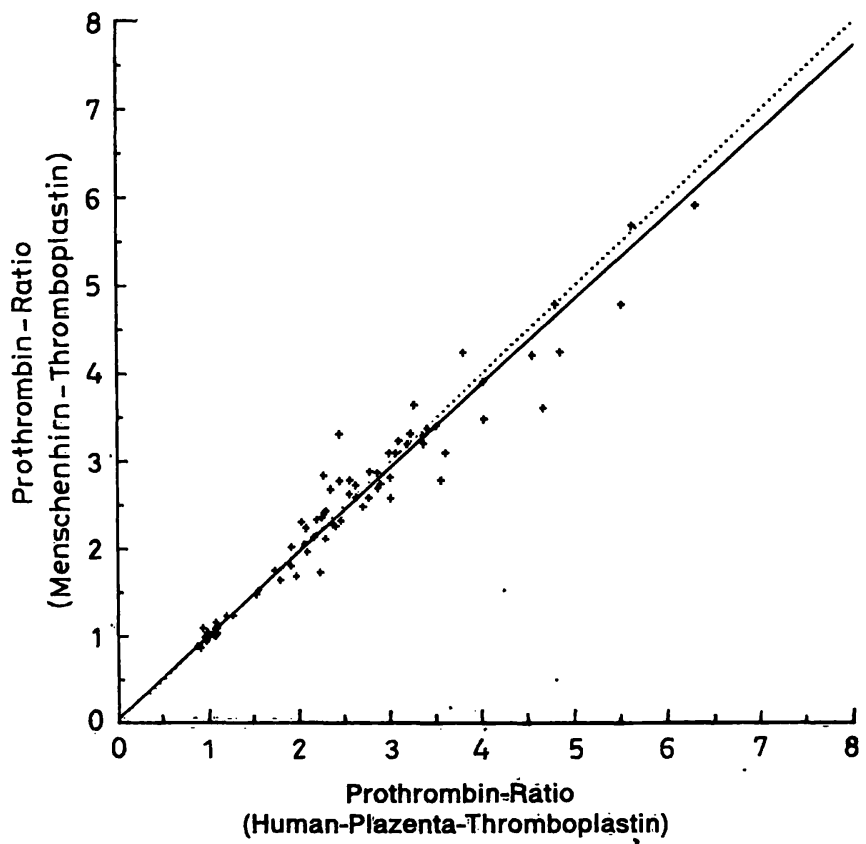

Abb. 11. Vergleichsuntersuchungen żwischën Thromboplastinzeit-Bestimmung mit dem Humañ-Plazenta-Thromboplastin und Menschenhirn-Thrombokinase (Basel) an einem Kollektiv von 80 Patienten.

Auswertungsmethode nach Passing \& Bablok (24): $y=0,96 x+0,05$

Keine signifikante Abweichung der Steigung von 1: 0,92 bis 1,01 .

Keine signifikante Abweichung des y-Achsenabschnitts von $0:-0,03$ bis 0,11 .

Methode der linearen Regression: $r=0,974$;

$y=0,91 x+0,18$ des Human-Plazenta-Thromboplastins anhand zweier Plasmapools von 150 Gesunden und von 40 stabil antikoagulierten Patienten (Thromboplastinzeit: $15-20 \%$ der Norm) an einem internen Thromboplastinstandard kalibriert, der gegen ein Referenzthromboplastin (British Comparative Thromboplastin Charge Nr. 235) vorkalibriert wurde. Für jede Thromboplastincharge wird nach Auftragung der Thromboplastinzeiten der zwei Plasmapools (je 36 Bestimmungen) mit dem Routine- und dem Referenzthromboplastin auf doppelt-logarithmischer Skala die Steigung (ISI = International Sensitivity Index = Internationaler Empfindlichkeitsfaktor) der Verbindungslinie berechnet (27).

Der internationale Empfindlichkeitsfaktor erlaubt über die Prothrombin-Ratio (PR) der antikoagulierten Patienten die reagenzienunabhängige und allgemein vergleichbare „International Normalized Ratio“ (INR) zu berechnen: INR $=\mathrm{PR}^{\text {ISI }}$ (28).

Je mehr sich der internationale Empfindlichkeitsfaktor eines Thromboplastins dem des WHO-Standards annähert, desto empfindlicher verhält sich dieses Thromboplastin bei der Überwachung der oralen Antikoagulantientherapie (29). Die Berechnung der Werte des internationalen Empfindlichkeitsfaktor für 14 Chargen des Human-Plazenta-Thromboplastins 
ergab einen Mittelwert von 1,16 mit einer Standardabweichung von 0,06 (Variationskoeffizient: 4,9\%) (Tab. 2).

Die vorläufige interne Standardisierung wurde im Rahmen der kooperativen Studie überprüft. Die Ergebnisse der Berechnung des internationalen Empfindlichkeitsfaktors nach der Methode der orthogonalen Regression (27) in jedem an der kooperativen Studie beteiligten Zentren sind in den Abbildung 12 wiedergegeben. Es wurden für den internationalen Empfindlichkeitsfaktor Werte von 1,09, 1,03, 1,09 und 1,07 ermittelt.
Nach Zusammentragung aller in den fünf Zentren gemessenen Thromboplastinzeit-Werte ergab sich nach der Methode der orthogonalen Regression als Internationaler Empfindlichkeitsfaktor für die in der Studie verwendete Human-Plazenta-Thromboplastin-Charge Nr. 110583 ein Wert von 1,08 (Abb. 13).

Die Ergebnisse dieser Studie erlauben die Etablierung eines sekundären Standards, der dann zur Kalibrierung aller neuen Thromboplastin-Chargen herangezogen werden kann.
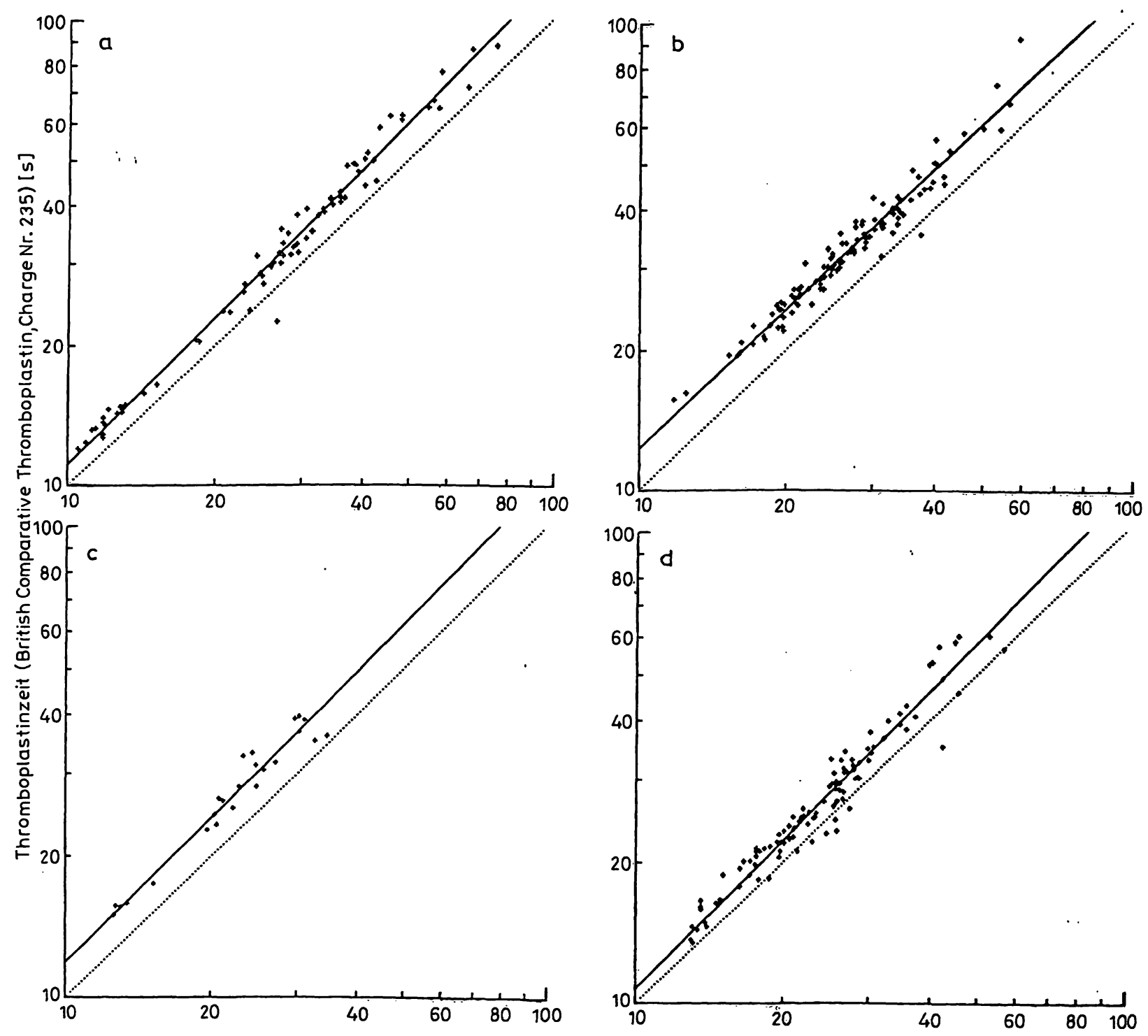

Thromboplastinzeit (Human-Plazenta-Thromboplastin) [s]

Abb. 12. Ermittlung des „International Sensitivity Index (ISI)“ für das Human-Plazenta-Thromboplastin gegen das British Comparative Thromboplastin Charge Nr. 235 in vier der an der kooperativen Studie beteiligten Zenitren: Auswertungsmethode: orthogonale Regression (27) (doppelt-logarithmische Skala).
a) $\mathrm{n}=80 ; \log (\mathrm{y})=1,05 \log (\mathrm{x})-0,01 ;$ ISI: 1,09
b) $\mathrm{n}=116 ; \log (\mathrm{y})=0,99 \log (\mathrm{x})+0,10 ;$ ISI: 1,03
c) $n=106 ; \log (y)=1,05 \log (x)-0,02 ;$ ISI: 1,09
d) $n=24 ; \log (y)=1,03 \log (x)+0,05$; ISI: 1,07 


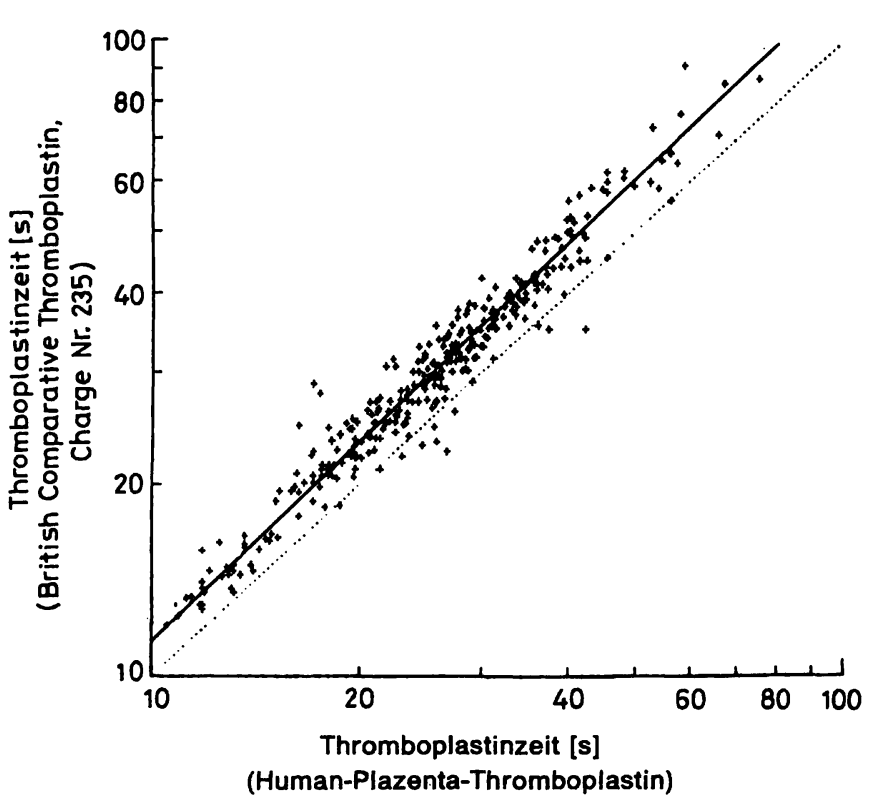

Abb. 13. Ermittlung des Internationalen Empfindlichkeitsfaktors (ISI) für den Behringwerke-Hausstandard Thromborel®S (Charge Nr. 110583) gegen die Referenzpräparation British Comparative Thromboplastin (Charge Nr. 235) (Int. Sensitivity Index: 1,039) aus den gesamten Ergebnissen dẹ kooperativen Studie $(n=330)$.

Auswertungsmethode: Orthogonale Regression (27) (doppelt-logarithmische Skala): $\log (y): 1,04 \log (x)+$ 0,$01 ;$ ISI: 1,08 .

Ermittlung des therapeutischen Bereichs des Human-Plazenta-Thromboplastins

Im Rahmen der kooperativen Studie konnte durch Verwendung speziell entwickelter Verfahren mittels eines Personal Computers der therapeutische Bereich des neuen Thromboplastins ermittelt werden. Dieses erfolgt durch die Heranziehung aller in den fünf Zentren durchgeführten Thromboplastinzeit-Bestimmungen, die gleichzeitig mit dem untersuchten Thromboplastin und mit dem British Comparative Thromboplastin Charge Nr. 235 durchgeführt wurden.

Mit Hilfe eines speziellen Rechnerprogramms ist es möglich, eine automatisierte Analyse der relativen Übereinstimmung für die Thromboplastinzeit-Werte, die sich im therapeutischen Bereich des British Comparative Thromboplastins und in einem variierenden Bereich des Human-Plazenta-Thromboplastins befinden, im Hinblick auf die Beurteilung der Antikoagulationsintensität vorzunehmen. Der Bereich für das untersuchte Thromboplaștin wird so lange variiert, bis die korrespondierenden ThromboplastinzeitWerte (d.h. die Thromboplastinzeit-Werte, die im selben Plasma mit zwei zu vergleichenden Thromboplastinen bestimmt wurden) die maximale Übereinstimmung in der Diagonale einer Neunfelder-Kontingenztafel ergeben. In Tabelle 3 wird die Neunfeldertafel zur Beurteilung der diagnostischen Übereinstim-
Tab. 3. Korrespondenzmatrix (Neunfelder-Kontingenztafel) für die Thromboplastinzeit-Werte oral antikoagulierter Patienten in \% der Norm für das British Comparative Thromboplastin und das Human-Plazenta-Thromboplastin zur Festlegung des therapeutischen Bereichs des Human-Plazenta-Thromboplastins. Der Bereich in $\%$ der Norm für das British Comparative Thromboplastin wurde aus den Prothrombin-Ratio-Werten abgeleitet.

British Comparative Thromboplastin (Prothrombin-Ratio)

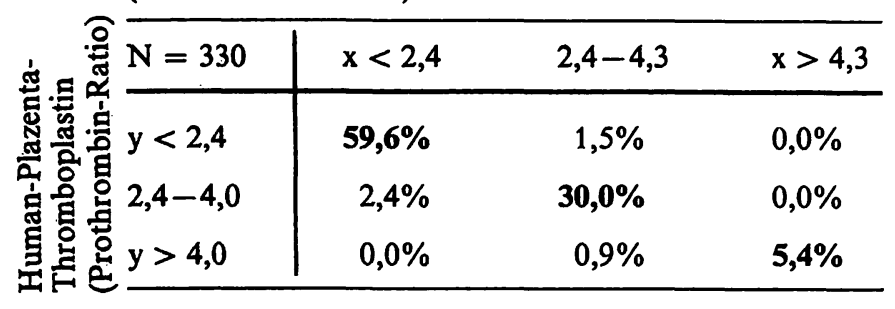

mung zwischen dem Human-Plazenta-Thromboplastin und dem Vergleichsthromboplastin British Comparative Thromboplastin bei Festlegung des therapeutischen Bereichs für Charge Nr. 235 des British Comparative Thromboplastins mit 2,4-4,3 Prothrombin-Ratio gèzeigt.

Dieser Wert entspricht einem Zielbereich von 2,5-4,5 International Normalized Ratio, der sowohl für die Prophylaxe der sekundären venösen Thrombose als auch bei der Behandlung von Herzinfarktpatienten angestrebt werden soll.

Die maximale Übereinstimmung, d.h. 95\%, wurde bei einem therapeutischen Bereich des Human-Plazenta-Thromboplastins von 2,4-4,0 ProthrombinRatio erhalten. Der Vergleich der Therapiebeurteilung mit Human-Plazenta-Thromboplastin und dem British Comparative Thromboplastin in \% der Norm (Tab. 4). ergab eine Übereinstimmung von $94,4 \%$ bei Verwendung eines therapeutischen Bereiches für das Human-Plażenta-Thromboplastin von 15 bis $27 \%$ der Norm.

Tab. 4. Festlegung des therapeutischen Bereiches für das Human-Plazenta-Thromboplastins. Korrespondenzmatrix für die Prothrombin-Ratio-Werte des British Comparative Thromboplastins und des Human-PlazentaThromboplastins. Die International Normalized Ratio des British Comparative Thromboplastins; 2,5-4,5 entspricht einer Prothrombin-Ratio des British Comparative Thromboplastins: $2,4-4,3$.

British Comparative Thromboplastin (\% der Norm)

\begin{tabular}{|c|c|c|c|}
\hline $\mathbf{N}=330$ & $x<14$ & $14-26$ & $x>26$ \\
\hline$y<15$ & $4,5 \%$ & $1,2 \%$ & $0,0 \%$ \\
\hline $15-27$ & $1,2 \%$ & $30,6 \%$ & $1,2 \%$ \\
\hline$y>27$ & $0,0 \%$ & $1,8 \%$ & $59,3 \%$ \\
\hline
\end{tabular}




\section{Diskussion}

Die Ergebnisse der kooperativen Studie zeigen, daß das neue Human-Plazenta-Thromboplastin eine gute Empfindlichkeit für alle Faktoren des exogenen $\mathrm{Ge}$ rinnungssystems einschließlich Faktor VII besitzt.

Die Empfindlichkeit des Human-Plazenta-Thromboplastins gegen Heparin - gemessen in in-vitro Untersuchungen - (0,6 IU Heparin pro ml Plasma) ist relativ gering, obwohl das Reagenz keinen zugesetzten Heparininhibitor enthält. Das erlaubt auch in der Einstellphase der oralen Antikoagulation, bei welcher Heparinkonzentrationen von 0,2 bis $0,5 \mathrm{IU} / \mathrm{ml}$ auftreten, anhand des Human-Plazenta-Thromboplastins noch gut die Thromboplastinzeit messen zu können. Andererseits würden jedoch gefährliche Überdosierungen über $0,7 \mathrm{IU} / \mathrm{ml}$ durch das Reagenz angezeigt werden.

Hohe Faktoren- und niedrige Heparinempfindlichkeit sind Eigenschaften, die eine wichtige Voraussetzung für die' Eignung des untersuchten Thromboplastins zur Bestimmung der Thromboplastinzeit im Rahmen der Kontrolle der oralen AntikoagulantienTherapie darstellen.

Die Konstanz der verschiedenen Chargen des $\mathrm{Hu}-$ man-Plazenta-Thromboplastins ist durch das spezielle Herstellungsverfahren gewährleistet. Durch die Verwendung von chargen- und gerätespezifischen $\mathrm{Be}$ zugskurven werden weitere Fehlerquellen ausgeschaltet. Die Variationskoeffizienten der gemessenen Gerinnungszeit belegen die gute Reproduzierbarkeit der Thromboplastinzeit-Bestimmung mit Hilfe des $\mathrm{Hu}-$ man-Plazenta-Thromboplastins.

Als Grenze für die Entscheidung über die Durchführung eines operativen Eingriffs kann ein Thromboplastinzeit-Wert von $<75 \%$ der Norm bzw. $>1,2$ Prothrombin-Ratio betrachtet werden.

Die Überprüfung der Standardisierung der Thromboplastinzeit-Bestimmung mit dem neuen Human-Plazenta-Thromboplastin im Rahmen der kooperativen Studie unter Verwendung der Methode der orthogonalen Regression (27) hat eine gute Vergleichbarkeit mit dem British Comparative Thromboplastin ergeben. Als Wert des internationalen Empfindlichkeitsfaktors für die in der Studie verwendete Charge des Human-Plazenta-Thromboplastins wurde ein Wert von 1,08 ermittelt (Abb. 14). Jede Charge des hier überprüften Human-Plazenta-Thromboplastins wird nach den Empfehlungen des Internationalen Committee for Standardization in Haematology (ICSH) und des International Committee on Thrombosis and Haemostasis (ICTH) (26) mit dem in dieser Studie gegenüber dem British Comparative Thromboplastin
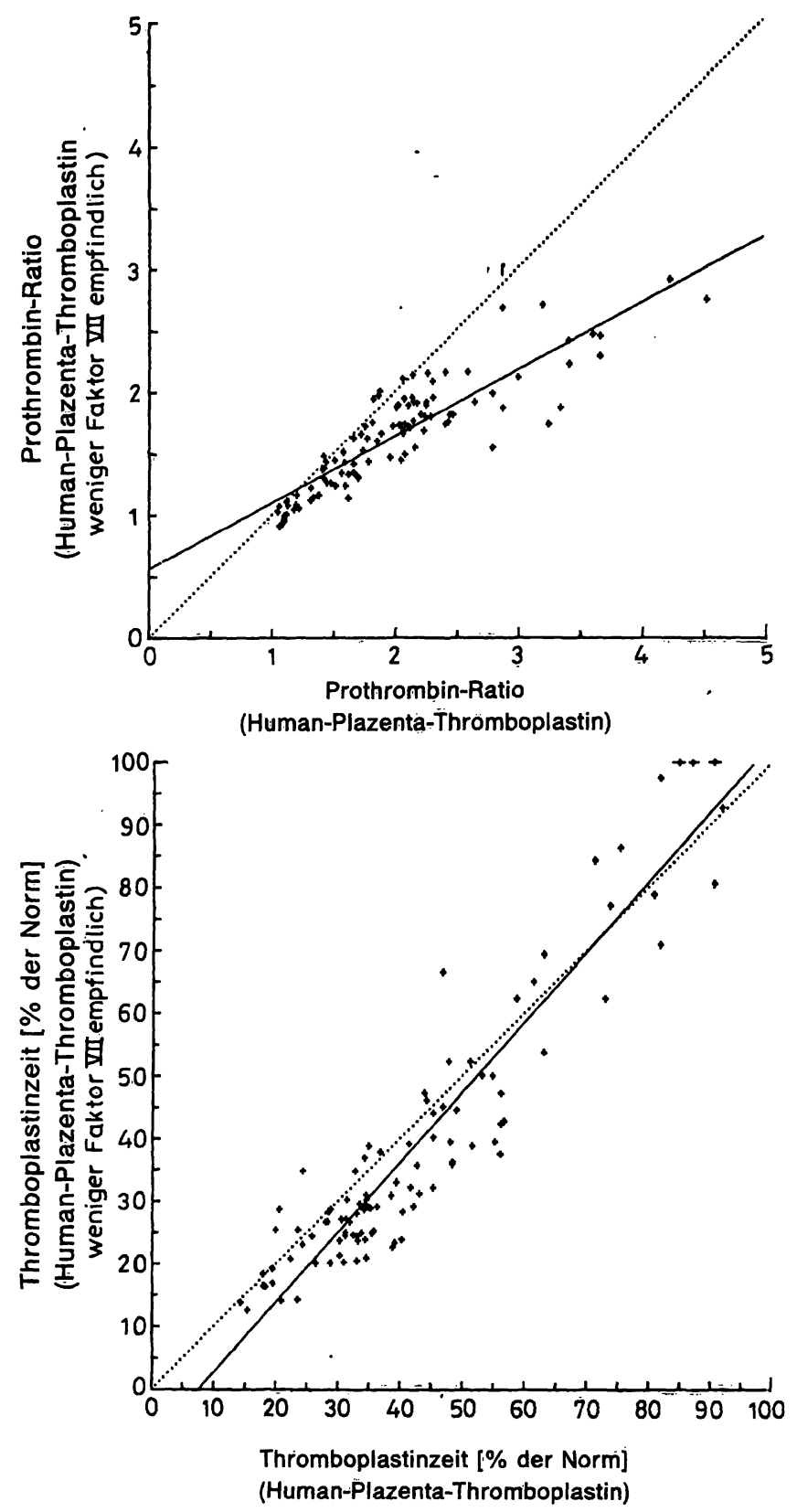

Abb. 14. Gegenüberstellung der Thromboplastinzeit-Werte oral antikoagulierter Patienten $(\mathrm{n}=106)$ in Prothrombin-Ratio und in \% der Norm für zwei in der Faktor VII-Empfindlichkeit unterschiedliche Thromboplastine.

Methode: lineare Regression.

Werte in Prothrombin-Ratio: $r=0,883$;

$\mathrm{y}=0,54 \mathrm{x}+0,6$.

Werte in \% der Norm: $\mathrm{r}=0,941 ; \mathrm{y}=1,12 \mathrm{x}-8,4$.

kalibrierten Human-Plazenta-Thromboplastin-Standard in Beziehung gebracht.

Die Standardisierung erfolgt anhand dieses internen Thromboplastinstandards mit Plasmen von Gesunden und von antikoagulierten Patienten und beinhaltet für jede Charge die Angabe vor einem für die Charge spezifischen Wert für den internationalen Empfindlichkeitsfaktor und den Ausdruck einer Tabelle, aus der „International Normalized Ratios“ entnommen werden können (Tab; $\left.{ }_{; i} 1\right)$. 
Die Ergebnisse der gesamten Vergleichsuntersuchungen zwischen dem Human-Plazenta-Thromboplastin und dem British Comparative Thromboplastin an oral antikoagulierten Patienten zeigen über den gesamten Bereich eine sehr gute Korrelation sowohl in Prothrombin-Ratio (Abb. 7) als auch in \% der Norm (Abb. 8) bei Anwendung der Methode der linearen Regression.

Da diese Methode aber gewisse Unzulänglichkeiten aufweist, besonders wenn keine Normalverteilung der untersuchten Werte vorliegt, wurde in dieser Studie auch das neue nicht-parametrische Verfahren nach Passing \& Bablok $(24,25)$ herangezogen. Dieses Verfahren zeichnet sich durch folgende Eigenschaften aus:

- Eine Normalverteilung der Meßwertepaare ist keine Voraussetzung.

- Der Verteilungstyp der Mẹfehler beider Methoden spielt eine untergeordnete Rolle.

- Die Methode ist auch bei Wertepaaren mit extremen Differenzen anwendbar; eine Ausreißerelimination ist daher nicht erforderlich.

- Die Linearität der Methoden wird im Rahmen des Verfahrens überprüft.

Bei einer solchen Korrelationsprüfung beeinflussen die stärker abweichenden Thromboplastinzeit-Werte im oberen Bereich die Steigung der Regressionsgeraden in geringerem Maße als es bei der Methode der linearen Regression der Fall wäre. Die gute Übereinstimmung der mit beiden Thromboplastinen gemessenen Thromboplastinzeit-Werte kann am besten festgestellt werden, wenn diese Werte als ProthrombinRatios ausgedrückt werden (Abb. 9). In der Tat zeigt die Korrelationsgerade der Prothrombin-Ratio-Werte keinen signifikanten Ünterschied zwischen beiden Methoden.

Der entscheidende Vorteil der Verwendung der Prothrombin-Ratio für dẹn Verglẹich der mit verschiedenen Thromboplastinen gemessenen Thromboplastinzeit-Werte wird besonders deutlich, wenn zwei in der Faktor VII-Empfindlichkeit unterschiedliche Thromboplastine verglichen werden. Aus der Darstellung (Abb. 14) der Thromboplastinzeit-Werte in Prothrombin-Ratio und in \% der Norm ist zu ersehen, daß die \%-der-Norm-Werte eine akzeptable Korrelation zeigen, obwohl die Streuung der Werte relativ groß ist.

Erst bei der Gegenüberstellung der Prothrombin-Ratios zeigt sich, daß die mit dem neụen empfindlichen Human-Plazenta-Thromboplastin erhaltenen Prothrombin-Ratio-Werte höher liegen. Durch die Verwendung der Prothrombin-Ratio-Werte ist dann bei der oralen Antikoagulantien-Therapie eine bessere Feineinstellung der Patienten möglich.

Aus den gesamten Ergebnissen der Thromboplastinzeit-Bestimmung bei oral antikoagulierten Patienten mittels dem British Comparative Thromboplastin und dem Human-Plazenta-Thromboplastin in den fünf Zentren wurde unter Verwendung des Neunfeldertafeltests der therapeutische Bereich für das hier untersuchte Human-Plazenta-Thromboplastin ermittelt. Als Zielbereich für das Vergleichsthromboplastin British Comparative Thromboplastin (Internationaler Empfindlichkeitsfaktor: 1,039) wurde eine Prothrombin-Ratio von 2,4 bis 4,3 (das entspricht einer International Normalized Ratio von 2,5-4,5) zugrunde gelegt.

Dieser Bereich ist als Kompromiß unter den unterschiedlichen für verschiedene Patientenkategorien propagierten optimalen Bereichen der Thromboplastinzeit $(8,23,30,31)$ anzusehen. Er berücksichtigt die an die verschiedenen klinischen Situationen angepaßten therapeutischen Bereiche für eine optimale Antikoagulation, u.a. bei sekundärer venöser Thrombose, bei kardialer und arterieller Indikation $(30,32)$ und weder unter- noch überschreitet er den von der WHO empfohlenen maximalen therapeutischen Bereich von 1,5 bis 5,0 Internationalen Normalized Ratio (33).

Die maximale Übereinstimmung für den angestrebten therapeutischen Bereich einer optimalen Antikoagulation von 2,5-4,5 International Normalized Ratio ist im Rahmen der kooperativen Studie bei einem Bereich des Human-Plazenta-Thromboplastins (Charge Nr. 110583) von 2,4 bis 4,0 ProthrombinRatio erhalten worden.

Zusammenfassend kann festgestellt werden, da $\beta$ die Ergebnisse der kooperativen Studie eine gute Vergleichbarkeit des hier untersuchten Thromboplastins aus Plazenta mit Thromboplastinen aus humanem Hirn zeigten. Die erfolgte Standardisierung des Human-Plazenta-Thromboplastins an internationalen Referenzpräparationen ermöglicht eine zuverlässige Bestimmung der Thromboplastinzeit bei Patienten unter oraler Antikoagulation mit der Möglichkeit der Angabe von reagenzien-unabhängigen und besser vergleichbaren Werten.

\section{Danksagung}

Für die wertvollen Diskussionsbeiträge und Anregungen im Rahmen der Prüfung, für die statistische und graphische Auswertung der Ergebnisse der Prüfung sowie für die Unterstützung bei der Anfertigung des Manuskriptes sei Herrn Dr. $U$. Becker, Herrn Herchenhan, Herrn Gentzer, Frau Langhoff und Frau Heberle besonders gedankt. 


\section{Literatur}

1. Janson, T. L., Stormorken, H. \& Prydz, H. (1984) Haemostasis 14, 440-444.

2. Quick, A. J., Stanley-Brown, M. \& Bancroft, F. W. (1935) Amer. J. Med. Sci. 190, 501-511.

3. Quick, A. J. (1959) Circulation 19, 92-96.

4. Loeliger, E. A. \& Van Halem-Visser, L. P. (1979) Thromb. Haemostas. 42, 1115-1127.

5. Averdunk, R. \& Borner, K. (1970) Z. Klin. Chem. Klin. Biochem. 8, 263-268.

6. Exner, T., Rickard, K. A. \& Kronenberg, H. (1980) Pathology $12,559-566$.

7. Biggs, R. \& Denson, K. W. E. (1967) Br. Med. J. 1, 84.

8. Marti, P. \& Beck, E. A. (1983) Schweiz. Med. Wochenschr. 113, 1593-1597.

9. Averdunk, R. \& Borner, K. (1974) Blut 28, 445-451.

10. International Committee on Thrombosis and Haemostasis and International Committee for Standardization in Haematology (1979) Thromb. Haemostas. 42, 1073-1114.

11. Kirkwood, T. B. L. (1983) Thromb. Haemostas. 49, $238-244$.

12. Poller, L. (1975) Thrombos. Diathes. Haemorrh. 33, $32-50$.

13. Poller, L. (1967) Lancet $I, 491-493$.

14. Thomson, J. M. \& Chart, I. S. (1970) J. Med. Lab. Technol. 27, 207-212.

15. WHO Expert Committee on Biological Standardization. 28th Report. WHO Technical Report Series 610 (1977), $14-15$ and $45-51$.

16. WHO Expert Committee on Biological Standardization. 33rd Report. WHO Technical Report Series 687 (1983), $81-105$.

17. van den Besselaar, A. M. H. P. (1985) Haemostasis 15, $271-277$.

18. Fuhge, P., Herchenhan, B., Dati, F., Becker, U. \& Heimburger, N. (1985) Haemostasis 15, 73.

19. Dati, F., Fuhge, P., Becker, U. \& Heimburger, N. (1985) Lab. Med. 9, 153.
20. Hermans, J., van den Besselaar, A. M. H. P., Loeliger, E. A. \& van der Velde, E. A. (1983) Thromb. Haemostas. $50,712-717$.

21. Kirkwood, T. B. L. (1984) in: Thromboplastin Calibration and Oral Anticoagulant Control (van den Besselaar, A. M. H. P., Grabnick, H.R. \& Lewis, S. M., Eds.), Martinus Nijhoff Publishers, Boston, pp. 1i1-23.

22. International Federation of Clinical Chemištry (IFCC) (1983) J. Clin. Chem. Clin. Biochem. 21, 749-760.

23. Loeliger, E. A., Meuwisse-Braun, J. B., Muis, H., Buitendij, F. J. J., Weetkamp, J. J. \& Hemker, H. C. (1970) Thrombos. Diathes. Haemorrh. 23, 569-584.

24. Passing, H. \& Bablok, W. (1983) J. Clin. Chem. Clin. Biochem. 21, 709-720.

25. Eisenwiener, H.-G., Bablok, W., Bardorff, W., Bender, R., Markowetz, D., Passing, H., Spaethe, R., Specht, W. \& Völkert, E. (1984) Lab. Mèd. 8, 232-244.

26. International Committee for Standardization in Haematology - International Committee on Thrombosis and Haemostasis (1985) Thromb. Haemostas. 53, 155-156.

27. van der Velde, E. A. (1984) in: Thromboplastin Calibration and Oral Anticoagulant Control (van den Besselaar, A. M. H. P., Grabnick, H.R. \& Lewis, S. M., eds.), Martinus Nijhoff Publishers, Boston, pp. 25=39.

28. Beck, E. A. (1985) Schweiz. Med. Wochenșchr. 115, $1378-1380$.

29. Loeliger, E. A., van den Bessselaar, A. M. H. P. \& Lewis, S. M. (1985) Thromb. Haemostas. 52, 148.

30. Loeliger, E. A. \& Lewis, S. M. (1982) Lancet II, 318-320.

31. Duckert, F. \& Marbert, G. A. (1977) Schweiz. Med. Wochenschr. 107, 1308.

32. Loeliger, E. A. (1985) Schweiz. Med. Wochenschr. 115, 1483-1495.

33. Thomson, J. M., Tomeson, J. A. \& Poller, L. (1984) Thromb. Haemostas. 52, 336-342.

Für die Verfasser:

Dr. Francesco Dati

Behringwerke AG

Postfach 1140

D-3550 Marburg 